\title{
APEX: A Prime EXperiment at Jefferson Lab
}

\section{Test Run Results and Full Run Plans; Update}

\author{
James Beacham ${ }^{1, a}$ \\ ${ }^{1}$ The Ohio State University, Department of Physics, 191 West Woodruff Ave Columbus OH, 43210
}

\begin{abstract}
APEX is an experiment at Thomas Jefferson National Accelerator Facility (JLab) in Virginia, USA, that searches for a new gauge boson $\left(A^{\prime}\right)$ with sub-GeV mass and coupling to ordinary matter of $g^{\prime} \sim\left(10^{-6}-\right.$ $\left.10^{-2}\right) e$. Electrons impinge upon a fixed target of high-Z material. An $A^{\prime}$ is produced via a process analogous to photon bremsstrahlung, decaying to an $e^{+} e^{-}$pair. A test run was held in July of 2010, covering $m_{A^{\prime}}=175$ to $250 \mathrm{MeV}$ and couplings $g^{\prime} / e>10^{-3}$. A full run is approved and will cover $m_{A^{\prime}} \sim 65$ to $525 \mathrm{MeV}$ and $g^{\prime} / e>2.3 \times 10^{-4}$, and is expected to occur sometime in 2016 or 2017.
\end{abstract}

\section{Motivations}

The Standard Model (SM) of particle interactions is described by an $\mathrm{SU}(3)_{C} \times \mathrm{SU}(2)_{L} \times \mathrm{U}(1)_{Y}$ gauge group, where the forces are mediated by vector bosons. For an extension of this model to have thus far evaded detection the corresponding gauge boson must have a mass of $O(\mathrm{TeV})$ or must be very weakly coupled to ordinary matter, with a coupling strength $g^{\prime}$ suppressed relative to the electromagnetic charge $e$ by $\epsilon \equiv g^{\prime} / e \sim 10^{-6}-10^{-2}$ [1] (or, equivalently, $\alpha^{\prime} / \alpha=\epsilon^{2}$ ). This new gauge boson, $A^{\prime}$, corresponding to a $\mathrm{U}(1)^{\prime}$ extension of the $\mathrm{SM}$ can acquire an effective interaction with electromagnetism via kinetic mixing, where quantum loops of arbitrarily heavy particles provide a means by which the hidden $\mathrm{U}(1)^{\prime}$ sector couples to the visible sector; see, e.g., [2-4]. The possibility of the existence of an $A^{\prime}$ with a small EM charge can be tested at fixed target facilities such as the Thomas Jefferson National Accelerator Facility (JLab). APEX, The A Prime EXperiment, searches for an $A^{\prime}$ at JLab and is described in brief here. For a full description of the experiment see [5] and for a more detailed description of the results of the test run see [6].

In addition to the general interest in discovering an extension of the SM, a hidden gauge sector with a gauge boson with mass in the $\mathrm{MeV}$ to $\mathrm{GeV}$ range could address dark matter anomalies and the anomalous magnetic moment of the muon. For a complete discussion of these possibilities, see [7].

\section{Existing Constraints}

Aside from these suggestive motivations, the coupling strength and mass of the $A^{\prime}$ are not predicted. Thus,

\footnotetext{
a e-mail: j.beacham@cern.ch
}

searches for this new gauge boson must be conducted over wide ranges of both. As a result, prior to 2009, the areas of parameter space probed by APEX were remarkably weakly constrained, since previous collider experiments that are sensitive to low mass particles would not have been able to collect large enough datasets to discover a particle with such a weak coupling to ordinary matter. Following the observation [7] that much of this range could be probed at existing experimental facilities, a renewed interest in such experiments has led to the current constraints and planned experimental sensitivities shown in Figure 1. For a complete description of these constraints, see [8] and references therein.

As seen in Figure 1, APEX covers a large portion of this area of parameter space, from $m_{A^{\prime}} \sim 65$ to $525 \mathrm{MeV}$ and with coupling reach to $g^{\prime} / e>2.3 \times 10^{-4}$. A test run for APEX was performed in July of 2010 and demonstrated the feasibility of the full experiment.

\section{APEX at Jefferson Lab's Hall A}

APEX is designed to take full advantage of JLab's Continuous Electron Beam Accelerator Facility and the two High Resolution Spectrometers (HRSs) in Hall A. For the test run, an electron beam of energy $2.260 \mathrm{GeV}$ and an intensity of up to $150 \mu \mathrm{A}$ was used, incident upon a tantalum foil of thickness $22 \mathrm{mg} / \mathrm{cm}^{2}$. The central momentum of each HRS was $\simeq 1.131 \mathrm{GeV}$ with a momentum acceptance of $\pm 4.5 \%$.

An $A^{\prime}$ is produced via a process analogous to photon bremsstrahlung and decays to an $e^{+} e^{-}$pair; thus, the $A^{\prime}$ signal will appear as a small, narrow bump in the invariant mass spectrum of $e^{+} e^{-}$pairs from background QED processes. The diagrams for signal and irreducible backgrounds are shown in Figure 2. 


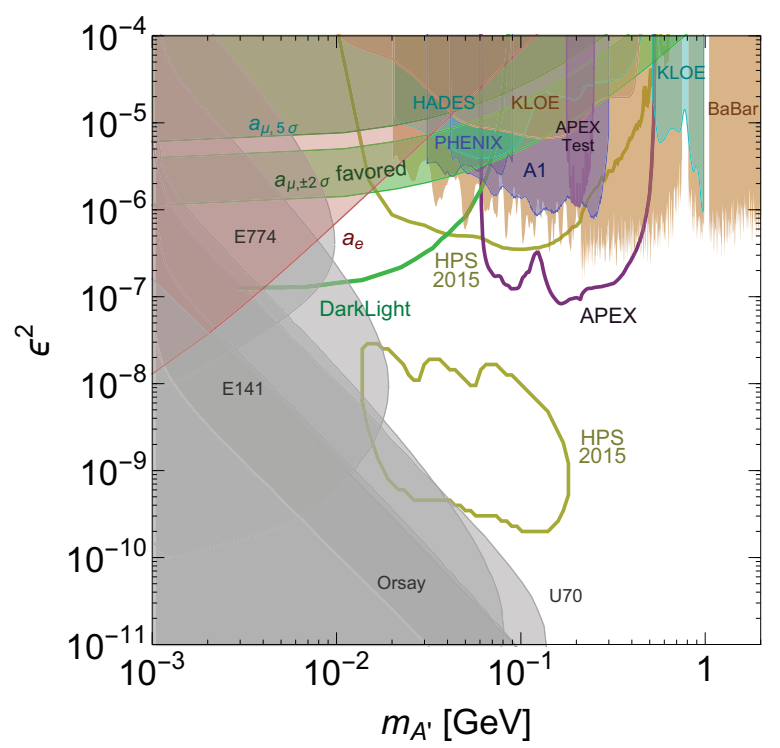

Figure 1: Existing and planned constraints in the $\epsilon-m_{A^{\prime}}$ plane, as of late 2014. From [9].

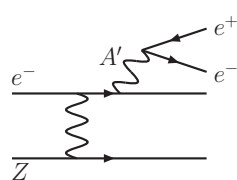

(a)

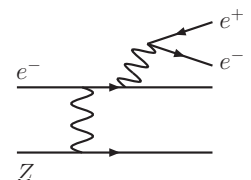

(b)

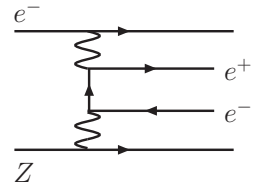

(c)
Figure 2: $A^{\prime}$ signal process (a) and irreducible QED backgrounds (b) and (c).

The opening angle $\Theta_{0}$ of the $e^{+} e^{-}$pair is set by $m_{A^{\prime}}$ and the incident electron beam energy as $\Theta_{0} \sim m_{A^{\prime}} / E_{b} \approx 5^{\circ}$, with no such expectation for the QED backgrounds. This motivates a symmetric HRS configuration with both spectrometer arms positioned far forward. To optimize sensitivity to $A^{\prime}$ decays, dipole septum magnets are placed between the target and the HRS aperture. A schematic overhead (side) view of this setup is shown in Figure 3 (4).

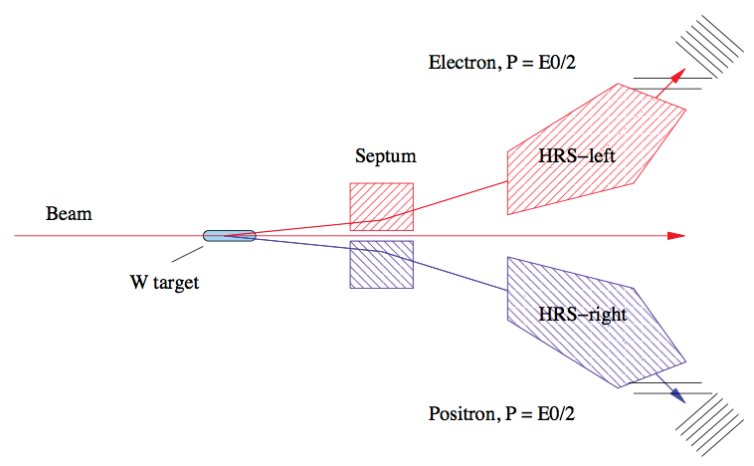

Figure 3: A schematic overhead view of JLab's Hall A, indicating septum magnets placed just past the target enclosure, to allow for the detection of $A^{\prime}$ decay products

(expected to have a very small opening angle).

The Hall A HRSs (Figure 5) consist of several different components to allow for the measurement of the posi-

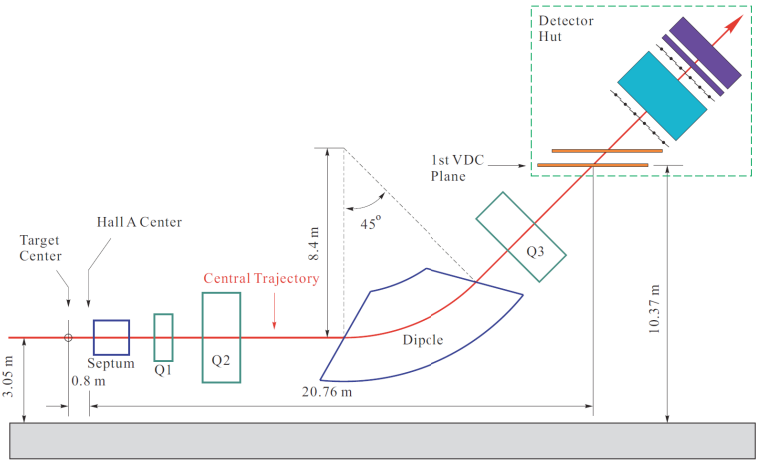

Figure 4: A schematic side view of the setup in JLab's Hall A.

tion and momentum of charged particles to a high degree of accuracy. Vertical drift chambers allow for an accurate determination of the full 3D track of an incoming particle. Two separate sets of scintillators provide timing information, to identify coincident $e^{+} e^{-}$pairs. Online particle ID is provided by a gas Cherenkov detector and a lead glass calorimeter allows for further offline rejection of pion backgrounds. A sieve-slit method is used for optics calibration.

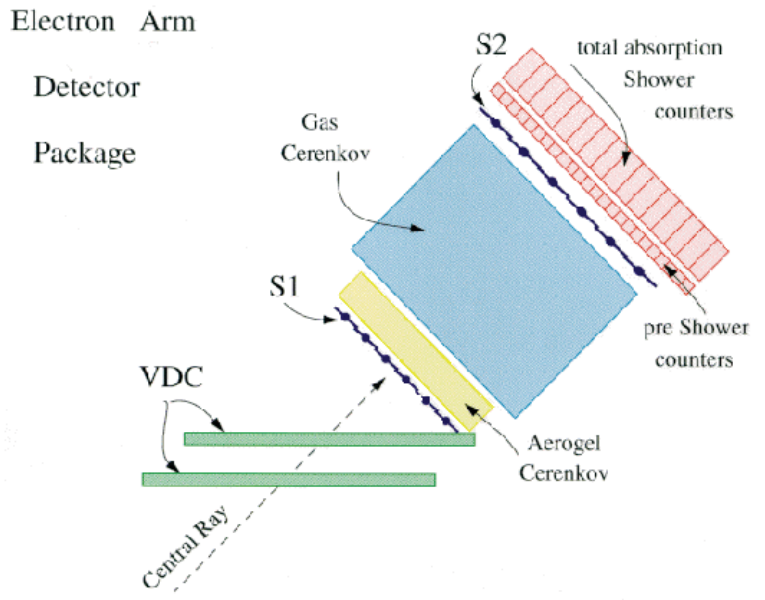

Figure 5: A schematic side view of electron-arm High Resolution Spectrometer (HRS) in JLab's Hall A. A similar setup is available in the positron arm.

Excellent mass resolution is required to enable the identification of an $A^{\prime}$ resonance. The HRSs are designed to achieve high momentum resolution at the level of $\delta p / p \sim 10^{-4}$, providing a negligible affect upon the mass resolution. Angular resolution and multiple scattering in the target are the dominant contributions to the mass resolution, as shown in Table 1. For the test run, APEX achieved a mass resolution of $\sigma \sim 0.85-1.11 \mathrm{MeV}$, varying over the full $m_{A^{\prime}}$ range.

Reducible backgrounds, including electron or proton singles, pions, accidental $e^{+} e^{-}$coincidences, and $e^{+} e^{-}$ 


\begin{tabular}{|c|c|c|c|}
\hline mrad & Optics & Tracking & MS in target \\
\hline$\sigma$ (horiz) & 0.11 & $\sim 0.4$ & 0.37 \\
\hline$\sigma$ (vert) & 0.22 & $\sim 1.8$ & 0.37 \\
\hline
\end{tabular}

Table 1: Contributions to APEX mass resolution.

pairs from real photon conversions, are rejected using a combination of different triggers.

The final event sample trigger for the test run required a double coincidence gas Cherenkov signal within a 12.5 ns window in each arm. The resulting data sample consisted of 770,500 true $e^{+} e^{-}$coincident events with $0.9 \%$ (7.4\%) meson (accidental $e^{+} e^{-}$coincidence) contamination.

\section{Resonance search}

The final data sample forms the basis of an invariant mass spectrum of $e^{+} e^{-}$pairs (shown in Figure 6), which is the starting point for the $A^{\prime} \rightarrow e^{+} e^{-}$search. Since the $A^{\prime}$ is expected to appear as a small, narrow bump on top of a smooth spectrum of background processes, a resonance search (also known as a raster scan or bump hunt) is performed to identify statistically significant excesses along the full mass spectrum.

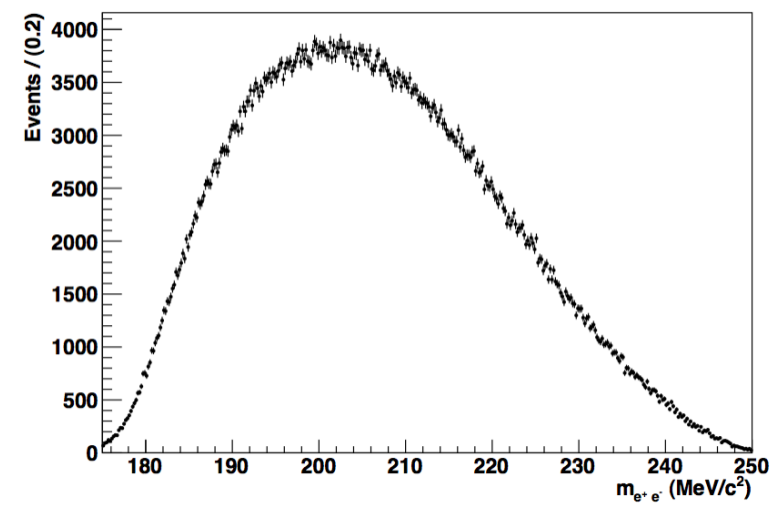

Figure 6: Distribution of $m_{e^{+} e^{-}}$, the result of the final selection for the APEX test run data.

In general, the procedure is as follows: One scans along the mass spectrum, and at each mass bin one hypothesizes a Gaussian peak of an unknown height and width $\sigma$ (also unknown but expected to be $O\left(\mathrm{MeV} / \mathrm{c}^{2}\right)$ ) equal to the experimental mass resolution. For a fixed $A^{\prime}$ mass $m_{A^{\prime}}$ and given hypotheses for the number of signal events $S$ and number of background events B, one models events as distributed according to a probability distribution given by

$$
\begin{aligned}
& P\left(m_{e^{+} e^{-}}\right)=\frac{1}{S+B}(S \cdot \mathrm{N}\left(m_{e^{+} e^{-}} \mid m_{A^{\prime}}, \sigma\right) \\
&\left.+B \cdot \operatorname{Polynomial}\left(m_{e^{+} e^{-}}, a_{i}\right)\right)
\end{aligned}
$$

where $m_{e^{+} e^{-}}$is the invariant mass of the electron-positron pair, $\mathrm{N}$ is a normal/Gaussian probability distribution, and the background shape is given by a polynomial with coefficients $a_{i}$. From the likelihood function, L, based on this probability model, we form a test statistic, $-2 \ln \lambda(\mathrm{S})$, where $\lambda(\mathrm{S})$ is the profile likelihood ratio (PLR),

$$
\lambda(S)=\frac{L\left(S, \hat{\hat{B}}, \hat{\hat{a}}_{i}\right)}{L\left(\hat{S}, \hat{B}, \hat{a}_{i}\right)}
$$

and where $\left.{ }^{\wedge} \hat{(}\right)$ indicates the unconditional (conditional) maximum likelihood estimator for that parameter, arrived at via fits of the model to the data. The systematic uncertainty in the background shape is incorporated into the profile likelihood ratio via the nuisance parameters $a_{i}$, the coefficients of the polynomial. An example of the results of the procedure of fitting the probability model to the data within one window (zoomed in to show detail) is shown in Figure 7. We then determine a confidence interval in number of signal events $S$, i.e., we determine the range in $\mathrm{S}$ consistent with the data at a given confidence level. The upper limit of this confidence interval corresponds to a limit on $\mathrm{S}$.

For the specifics of the analysis of the APEX test run data, based on extensive simulated-experiment studies, a 7 th-order polynomial fit over a $30.5 \mathrm{MeV}$ window is found to strike a near optimal balance between the simultaneous needs to maximize sensitivity to a signal and minimize pull (defined as $\sigma_{S \text {, bestfit }} / S_{\text {bestfit }}$ ) across the full mass spectrum; see Figures 8 and 9.

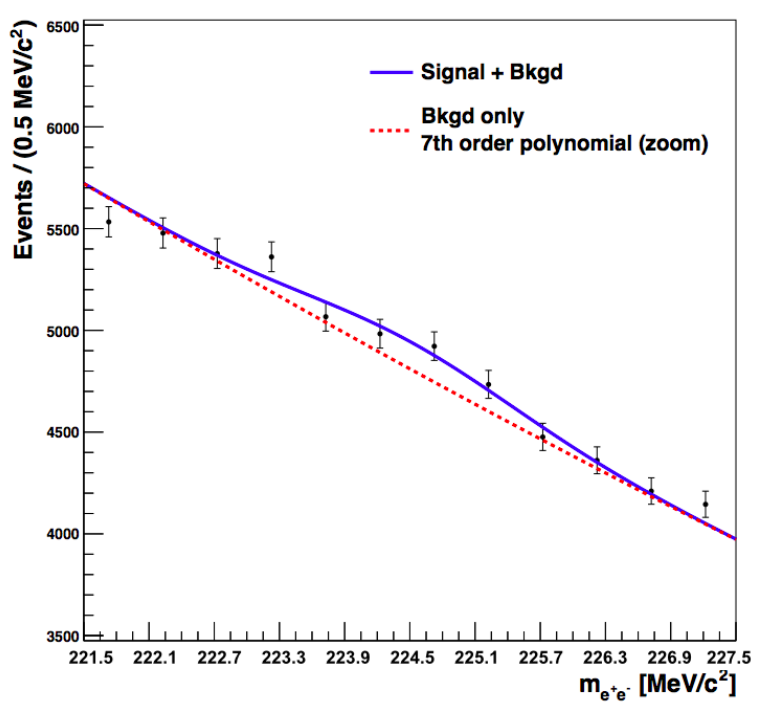

Figure 7: Detail of an example window for the resonance search.

A symmetric scanning window is used, except for candidate $A^{\prime}$ masses within $15 \mathrm{MeV}$ of the upper or lower boundaries, for which the window size is adjusted to correspond to twice the distance from the candidate mass to the boundary. The binned PLR described above is computed as a function of number of signal events $S$ at the candidate mass, using $0.05 \mathrm{MeV}$ bins. The PLR is used to derive the local probability ( $p$-value) at $S=0$ (i.e. the probability of a larger PLR arising from statistical fluctuations 


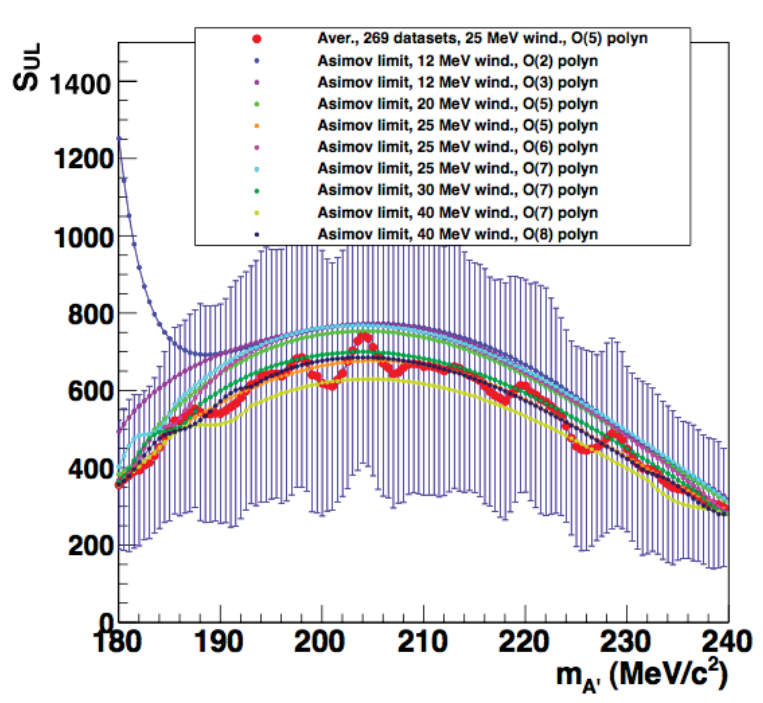

Figure 8: Upper limit on number of signal events, $S$ for pseudodatasets and Asimov data, for different window sizes and polynomial orders. The optimal values are a 30 $\mathrm{MeV}$ window and 7 th order polynomial.

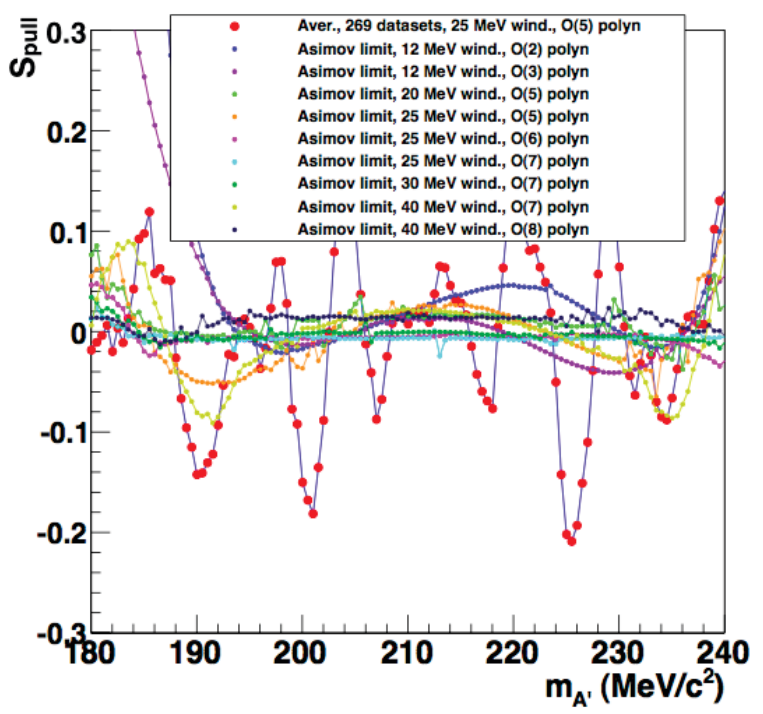

Figure 9: Signal pull for pseudodatasets and Asimov data, for different window sizes and polynomial orders. The optimal values are a $30 \mathrm{MeV}$ window and 7 th order polynomial.

in the background-only model) and a 90\%-confidence upper limit on the signal. The sensitivity of the search is defined in terms of a $50 \%$ power-constraint [10], which means we do not regard a value of $S$ as excluded if it falls below the expected limit. This procedure is repeated in steps of $0.25 \mathrm{MeV} / \mathrm{c}^{2}$. A global $p$-value, corrected for the "look-elsewhere effect", (the fact that an excess of events anywhere in the range can mimic a signal), is derived from the lowest local $p$-value observed over the full mass range, and calibrated using simulated experiments.

\section{Test Run Results}

No significant excess was found over the invariant mass range of $m_{A^{\prime}}=175$ to $250 \mathrm{MeV}$; see Figure 10 . The most significant excess was at $224.5 \mathrm{MeV}$ with a p-value of $0.06 \%$. Out of $\sim 1000$ pseudoexperiments based on the test run data, $40 \%$ yielded a p-value at least as extreme as $0.06 \%$ somewhere in the mass range.

The upper limit on number of signal events, $S$, compatible with a background fluctuation at the $90 \%$ CL was translated into an upper limit on the $A^{\prime}$ coupling, $\alpha^{\prime} / \alpha$, by exploiting the kinematic similarities between $A^{\prime}$ and $\gamma^{*}$ production [7]. Based on Monte Carlo simulations, the ratio $f$ of the radiative-only cross section to the full QED background cross section varies linearly from 0.21 to 0.25 across the APEX mass range and, thus, all backgrounds can be normalized to the radiative background. The final expression relating $S_{\max }$ and $\left(\alpha^{\prime} / \alpha\right)_{\max }$ is

$$
\left(\frac{\alpha^{\prime}}{\alpha}\right)_{\max }=\left(\frac{S_{\max } / m_{A^{\prime}}}{f \cdot \Delta B / \Delta m}\right) \times\left(\frac{2 N_{\mathrm{eff}} \alpha}{3 \pi}\right),
$$

where $N_{\mathrm{e} f f}$ counts the number of available decay channels $\left(N_{\mathrm{e} f f}=1\right.$ for $m_{A^{\prime}}<2 m_{\mu}$, and increases to $\simeq 1.6$ at $m_{A^{\prime}} \simeq 250 \mathrm{MeV}$ ). The upper limit on coupling is shown in Figure 11. A schematic view of the approach by which $S$ and $B$ are calculated for each mass hypothesis is shown in Figure 12.

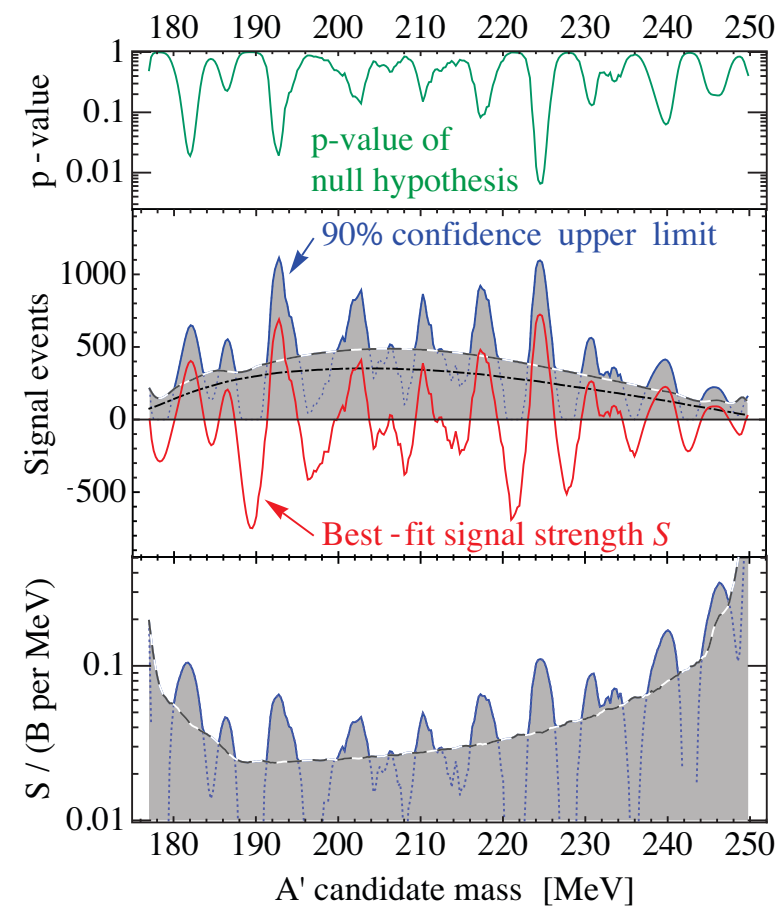

Figure 10: Results of the resonance search.

The data from the test run, in the form of the invariant mass values of the $e^{+} e^{-}$pairs, are publicly available [11].

\section{Full Run Plans}

The APEX full run is approved and is currently ready to run as an alternate experiment in JLab's Hall A sometime 


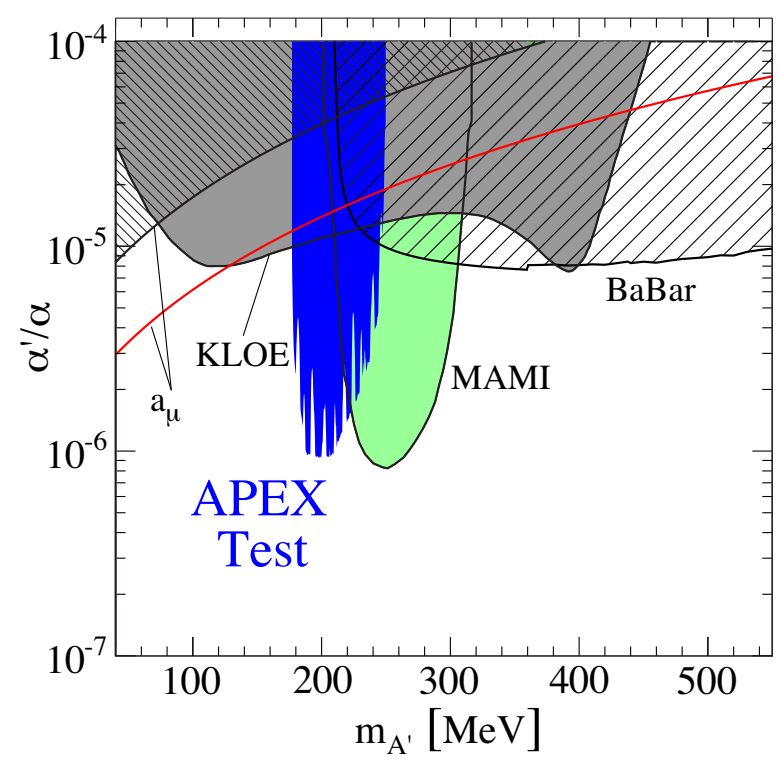

Figure 11: Upper limit on coupling.

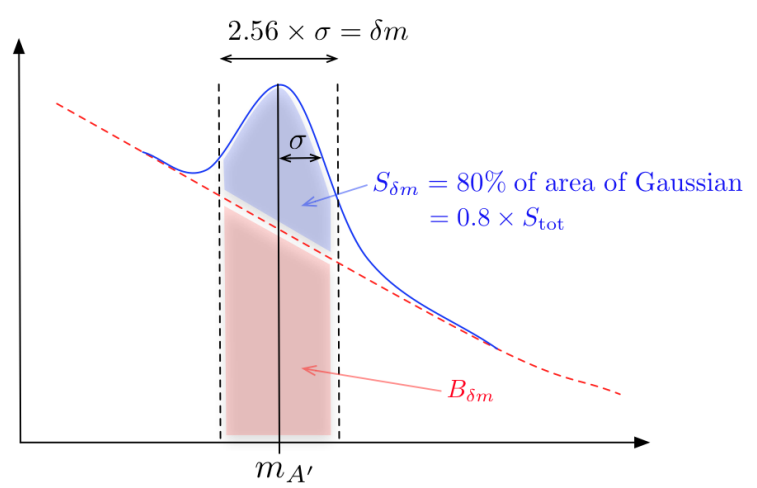

Figure 12: A schematic of the approach to determining number of signal events, $S$, over number of background events, $B$, in a resonance search.

during 2016 or 2017 . The full run will take data for $\sim 34$ days at four different energy and spectrometer settings, and will cover a larger mass range, $m_{A^{\prime}}=65$ to $525 \mathrm{MeV}$, using a $50 \mathrm{~cm}$ long multifoil target. The statistics of the full run will be $\sim 200$ times larger than the test run, allowing sensitivity to $\alpha^{\prime} / \alpha 1-2$ orders of magnitude below current limits. A complete description of the original plans for the full run is in [5].

A new optics calibration method, using a scintillating fiber hodoscope, is currently being tested. Additionally, data acquisition rates are being improved, to allow for up to $5 \mathrm{kHz}$. Moreover, a new septum magnet has been built and tested. The new septum magnet affects the acceptance of the APEX full run and, as such, the new field is currently being mapped and the changes in the acceptance propagated to the APEX sensitivity estimate; these results will appear soon. Additionally, possible modifications to the original run plan are being considered that could take advantage of higher beam energies and wider angles (with adjustment of the septum magnets) to access the higher $m_{A^{\prime}}$ region.

\section{Acknowledgments}

The author acknowledges Rouven Essig, Philip Schuster, Natalia Toro, Bogdan Wojtsekhowski, Sergey Abrahamyan, Eric Jensen, Jin Huang, Kyle Cranmer, and the rest of the members of the APEX collaboration. Additional thanks are due the Hall A collaboration and the Jefferson Lab staff for their outstanding support.

\section{References}

[1] R. Essig, P. Schuster and N. Toro, "Probing Dark Forces and Light Hidden Sectors at Low-Energy e+eColliders," Phys. Rev. D 80, 015003 (2009).

[2] B. Holdom, "Two U(1)'s and Epsilon Charge Shifts," Phys. Lett. B 166, 196 (1986).

[3] P. Galison and A. Manohar, "Two Z's or Not Two Z's?,’ Phys. Lett. B 136, 279 (1984).

[4] M. J. Strassler and K. M. Zurek, "Echoes of a hidden valley at hadron colliders," Phys. Lett. B 651, 374 (2007) [hep-ph/0604261].

[5] R. Essig, P. Schuster, N. Toro and B. Wojtsekhowski, "An Electron Fixed Target Experiment to Search for a New Vector Boson A' Decaying to e+e-," JHEP 1102, 009 (2011) [arXiv:1001.2557 [hep-ph]].

[6] S. Abrahamyan et al. [APEX Collaboration], "Search for a New Gauge Boson in Electron-Nucleus FixedTarget Scattering by the APEX Experiment," Phys. Rev. Lett. 107, 191804 (2011) [arXiv:1108.2750 [hep-ex]].

[7] J. D. Bjorken, R. Essig, P. Schuster and N. Toro, "New Fixed-Target Experiments to Search for Dark Gauge Forces," Phys. Rev. D 80, 075018 (2009) [arXiv:0906.0580 [hep-ph]].

[8] M. Endo, K. Hamaguchi and G. Mishima, "Constraints on Hidden Photon Models from Electron g-2 and Hydrogen Spectroscopy," Phys. Rev. D 86, 095029 (2012) [arXiv:1209.2558 [hep-ph]].

[9] R. Essig, J. Jaros, W. Wester, and others, "Dark Sectors and New, Light, Weakly-Coupled Particles," [arXiv:1311.0029 [hep-ph]].

[10] G. Cowan, K. Cranmer, E. Gross and O. Vitells, "Power-Constrained Limits," [arXiv:1105.3166].

[11] "Data from Figure 3 from: Search for a New Gauge Boson in Electron-Nucleus Fixed-Target Scattering by the APEX Experiment," Phys. Rev. Lett. 107, 191804 (2011) [arXiv:1108.2750 [hep-ex]]. 